Angew Chem Int Ed Engl. 2018 October 01; 57(40): 13242-13247. doi:10.1002/anie.201808823.

\title{
Discovery of Small Molecule Ligands for MALAT1 by Tuning an RNA-Binding Scaffold
}

\author{
Anita Donlic, Brittany S. Morgan, Jason L. Xu, Anqi Liu, Carlos Roble Jr., and Amanda E. \\ Hargrove* \\ Department of Chemistry, Duke University Durham, NC 27708-0346 (USA)
}

\begin{abstract}
Structural studies of the $3^{\prime}$-end of the oncogenic long non-coding RNA metastasis-associated lung adenocarcinoma transcript 1 (MALAT1) confirmed a unique triple-helix structure. This structure enables accumulation of the transcript, and high levels of MALAT1 are found in several cancers. Here, we synthesize a small molecule library based on an RNA-binding scaffold, diphenylfuran (DPF), screen it against a variety of nucleic acid constructs, and demonstrate for the first time that the MALAT1 triple helix can be selectively targeted with small molecules. Computational analysis revealed a trend between subunit positioning and composition on DPF shape and intramolecular interactions, which in turn generally correlated with selectivity and binding strengths. This work thus provides design strategies toward chemical probe development for the MALAT1 triple helix and suggests that comprehensive analyses of RNA-focused libraries can generate insights into selective RNA recognition.
\end{abstract}

\section{Keywords}

long noncoding RNA; molecular recognition; principal moment of inertia; RNA recognition; small molecule ligands

The recently renewed interest in small molecule :RNA targeting is in part due to the completion of the ENCODE project, which resulted in the discovery that the majority of the human transcriptome is non-protein-coding. ${ }^{[1]}$ Subsequent efforts to functionally characterize these non-coding transcripts resulted in the identification of novel RNA classes including long non-coding RNAs (lncRNAs). These molecules were found to have developmental- and tissue-specific expression and to regulate many levels of cellular processes, including expression of oncogenes. ${ }^{[2]}$ As a result, several lncRNAs have been proposed as therapeutic targets. ${ }^{[3]}$ As structured RNA elements are continually identified in these transcripts, utilizing small molecules to probe the specific functions and interactions of these domains represents an exciting avenue toward understanding ncRNA biology.

LncRNA metastasis-associated lung adenocarcinoma transcript 1 (MALAT1) has been recognized as a potential anticancer target due to its overexpression in cancerous tissues as

*amanda.hargrove@duke.edu.

Conflict of interest

The authors declare no conflict of interest. 
well as studies demonstrating that its knockdown reduces tumor growth and metastasis in preclinical models of various cancers. ${ }^{[4]}$ A study in colorectal cancer cells showed that a $\approx 1500$ n.t. segment at the evolutionarily conserved $3^{\prime}$-end of MALAT1 was sufficient to increase invasion and proliferation, implying that this region enables its oncogenic function. ${ }^{[5]}$ The recent structural characterization of a 74 n.t. region at the $3^{\prime}$-end of MALAT1 by Xray diffraction confirmed a unique, bipartite triple helix where the U-rich stem loop sequesters the A-rich tail, which is proposed to prevent exonucleolytic degradation (Figure 1a). ${ }^{[6]}$ Notably, the deletion of this segment decreased accumulation of the MALAT1 transcript. A comparable decrease in accumulation was also observed upon mutation of a Hoogsteen-positioned uridine, proposed to disrupt the triple-helix structure, indicating that subtle alterations in the stability of this structure can lead to significant changes in transcript level. The prospect of targeting this unique tertiary structure with small molecules therefore represents an attractive therapeutic strategy and a model system for exploring the druggability of lncRNA structural domains.

While several laboratories have recently reported small molecule probes for select mammalian RNA structures, newly discovered structural motifs in lncRNAs are emerging targets and remain largely underexplored with small molecule probes. ${ }^{[3]}$ Currently, there are no small molecule ligands reported to target the MALAT1 triple helix. The most closely related research has explored the targeting of simple T-A-T and U-A-U triplex structures with both promiscuous ligands (i.e. aminoglycosides ${ }^{[7]}$ and dyes ${ }^{[8]}$ ) and the tunable diphenylfuran (DPF)-based scaffold, furamidine. This scaffold has been diversified for preferential binding to a variety of nucleic acid structures, including various RNA and DNA duplexes, ${ }^{[9]} \mathrm{T}-\mathrm{A}-\mathrm{T}$ DNA triple helices, ${ }^{[10]}$ and disease-relevant stem-loops. ${ }^{[11]}$ Varied binding modes have been reported, including intercalation, groove binding and threading intercalation. ${ }^{[9 \mathrm{a}, 11 \mathrm{~b}]}$ An additional advantage of the DPF scaffold is its intrinsic fluorescence, which is quenched upon binding to RNA and thus enables evaluation of binding affinities without RNA or small molecule labeling. ${ }^{[11 \mathrm{~b}]}$ The tunability of this scaffold, its intrinsic fluorescent properties, as well as literature precedence for binding to a triple helix, position DPF as an excellent starting point for testing the susceptibility of a lncRNA triple helix toward targeting with a small molecule. In this study, we design and synthesize a library of DPF-based small molecules to identify a first-in-class selective ligand for the MALAT1 triple helix as well as obtain fundamental insights into the role of small molecule structure and shape in triple-helix binding strength and selectivity

We reasoned that a small molecule with the ability to selectively recognize and disrupt the triple helix could either directly displace the A-rich tail by binding to the stem-loop structure or stabilize a non-triple helical conformation. We thus began by evaluating the original DPF scaffold, furamidine, for binding to the MALAT1 triple helix and the stem loop construct, a structure without the triplex-forming A-rich tail (Figure $1 \mathrm{a}, \mathrm{b}$ ). The change in intrinsic fluorescence of the scaffold was measured upon increasing RNA concentrations, which enabled us to calculate the $\mathrm{EC}_{50}$ as an indicator of relative binding strengths as conducted previously. ${ }^{[9 \mathrm{~b}]}$ The comparison of this binding $\mathrm{EC}_{50}$ with the triple helix and stem loop RNA suggests that furamidine binds to the triple helix with $\approx 3$-fold selectivity as compared to the stem loop (Figure $1 \mathrm{c}$ ). Encouraged by this result, we envisioned a strategy of efficiently 
tuning the RNA-binding properties of the DPF scaffold by altering the composition and placement of subunits to achieve orthogonal recognition of each structure.

First, we identified and optimized a robust one-step coupling procedure to access all three symmetric regioisomers (para-, meta-, and ortho-) of the dinitrile DPF scaffold $\mathbf{3}$ (Scheme 1). ${ }^{[12]}$ To quickly diversify the scaffolds, we utilized eleven commercially available primary amine subunits enriched in heteroatoms and aromatic rings, chemotypes that are known to be important for selective RNA recognition. ${ }^{[13]}$ Subunit addition to the three dinitrile scaffolds 3 was achieved by optimizing previously reported copper ${ }^{[14]}$ - or aluminum ${ }^{[15]_{-}}$ assisted coupling reactions for diamidine formation to afford three sublibraries ( $\mathbf{p}, \mathbf{m}$, and $\mathbf{o})$ with eleven identical subunits on each scaffold (DPF-R). This final one-step coupling, which had not been applied to the synthesis of DPF-based small molecules, was found to increase efficiency relative to a reported two-step procedure that involves an imidate ester intermediate. ${ }^{[16]}$ To the best of our knowledge, this route thus represents the shortest synthesis of DPF-based libraries to date. Further, all of the synthesized library members are novel with the exception of DPFp1 and DPFm1. These latter ligands were strong binders of several therapeutically relevant RNAs, ${ }^{\left[{ }^{[a}, 11 c, 17\right]}$ therefore representing an adequate reference point for binding preferences of the newly synthesized ligands herein.

We next evaluated our DPF-based small molecule library for binding to the MALAT1 triple helix and stem loop RNAs via the aforementioned fluorescence-based screen. The para-DPF sublibrary contained the only library member (DPFp8) that demonstrated binding exclusively with the triple helix (Figure 2 a, Table S1 in the Supporting Information). Further, only this sublibrary contained ligands with $\mathrm{EC}_{50}$ values in the low nanomolar range, which were similar to the reported binding affinity of the A-rich tail for the stem loop $\left(\mathrm{K}_{\mathrm{d}} \approx\right.$ $20 \mathrm{nM}){ }^{[18]}$ Thermal melting studies of these DPFs resulted in stabilizing effects on the triple helix (Figure S1, Table S2), though we note these experiments require RNA and ligand concentrations that are well above the $\mathrm{EC}_{50}$ values in order to measure absorbance. All meta-substituted DPFs displayed $\mathrm{EC}_{50}$ values in the micromolar range and preferential binding for the triple helix in the fluorescence assays, which is in agreement with literature for the binding of other meta-substituted DPFs to a DNA triple helix. ${ }^{[10]}$ Nevertheless, we noted little variability in binding strengths and selectivities for the triple helix versus the stem loop across the different members of the meta-DPF sublibrary (Figure 2 a, Table S1). This observation is also supported by previous studies that reported lower selectivity between DNA duplexes for meta-as compared to para-substituted DPFs. ${ }^{[19]}$ Similarly, the $\mathrm{EC}_{50}$ values obtained for the ortho-DPF sublibrary indicated preferential binding for the triple helix over the stem loop, albeit with greater variability in selectivity between the two targets as compared to meta-DPF sublibrary (Figure 2 a, Table S1).

We distinguished one subunit-based trend that held for all three scaffolds. Namely, ligands with the benzyl-methylpiperidine subunit $\mathbf{8}$ were among the most selective molecules for the triple helix over the stem loop (Table S1). The remaining subunit-based trends were observed only for the para-DPF sublibrary. For example, we noted that aliphatic subunits with protonated nitrogens (DPFp1, -p9 and -p10) generally increase binding strength but do not confer much selectivity between the two targets. Presence of a chlorine moiety on the ortho-position of the benzene subunit (DPFp6 vs. DPFp4) was important for increasing 
binding strength towards the stem loop, while placement at the para-position (DPFp6 vs. DPFp5) reversed the selectivity between the targets (Figure 2a). Lastly, we noted that the only sulfur-containing moiety, the thiophene on DPFp11, allowed selective binding towards the stem loop.

To gain more insights into the possible subunit-based determinants of selectivity, seven paraDPF sublibrary members with varying subunit composition (DPFp1, -p4, -p6, -p8, -p9, p10, and -pll) were selected for further evaluations against nucleic acids of varying topologies. We evaluated the select library members for binding to: i) tRNA, which constitutes $\approx 20 \%$ of RNA in cells; ii) an AT-rich DNA duplex, a known target for DPFbased small molecules, and iii) the Rev-response element (RRE) stem loop IIB, a shorter stem-loop construct that was also evaluated for binding to various DPF scaffold-based libraries. ${ }^{[11 b, c, 17]}$ Our results show that the library members with aliphatic, positively charged nitrogen subunits (DPFp1, -p9 and -p10) bind to all RNA controls with similar $\mathrm{EC}_{50}$ values, indicating non-specific, likely electrostatic interactions with the RNA backbone (Figure 2b, Tables S3, S6, S8 and S9). On the contrary, library members with aromatic subunits (DPFp4, -p6 and -p11) appear to confer selectivity for the MALAT1 stem loop as reflected in statistically significant differences in their binding strength for this target over all other nucleic acids tested in this screen (Figure 2b, Tables S5, S6, and S10). Lastly, the only exclusive triple helix binder from our initial evaluations (DPFp8) displayed significantly weaker binding strengths for other constructs (Figure 2b, Table S7). It is worth noting that these experiments specifically capture binding events that modulate the fluorescence of the DPF scaffold and thus binding events that do not change fluorescence cannot be ruled out.

We next performed computational analyses to further understand the impact of subunit positioning on DPF scaffold tunability for differential recognition of MALAT1. We first assessed the 3D shape coverage of the three sublibraries (ortho, meta, para), as shape is known to be important for RNA recognition. ${ }^{[13 a, 20]}$ Specifically, we calculated principal moments of inertia (PMI), a spatial measurement that describes ligands as rod, disk, sphere or hybrid. ${ }^{[21]}$ First, a low energy ensemble of each ligand was generated via molecular dynamics simulations (MD) utilizing a generalized Born solvation model. PMIs were calculated for each structure in the ensemble, and the resulting coordinates were averaged for each ligand using the Boltzmann equation. Several clear trends were revealed in this analysis: i) the para- DPF sublibrary is exclusively in the rod sub-triangle, ii) the majority of the meta-DPF sublibrary is within the disk and hybrid sub-triangles, with the exception of four ligands in the rod sub-triangle and iii) the ortho-DPF sublibrary is distributed in the sphere, disk, and hybrid sub-triangles and is thus the least rod-like (Figure 3 a, Figure S2, Table S11).

Previous PMI calculations performed by our laboratory revealed that bioactive RNAtargeted ligands, which are assumed to be selective, have more rod-like character as compared to FDA-approved drugs or in vitro binding RNA ligands with no reported bioactivity. ${ }^{[13 a]}$ It was therefore of particular interest that the para-DPF sublibrary, which contained the strongest binders and the only triple helix-selective ligand, contained exclusively rod-shaped ligands. A closer look at this sublibrary revealed that most members 
had a similar shape except for DPFp7 and DPFp8. Specifically, DPFp7 had more disc-like character than the bulk of the library, and it is the only ligand in the para-DPF sublibrary that did not bind the triple helix or the stem loop. DPFp8 had the most rod-like character in the entire library and is the only library member that displayed exclusive selectivity for the triple helix.

In addition, the benzyl-piperidine subunit of DPFp8 is among the subunits that provides the most rod-like shapes for the ortho- and meta-sublibraries (Table S11) and is present in the ligands that conferred the highest selectivity for the triple helix over stem loop constructs in all three sublibraries (Table S1). Upon inspecting all conformations in the ensembles of these ligands, we noted extended shapes that appeared to be locked by the ring identity and connectivity of this subunit (Figure S3). Indeed, this subunit has been used in the literature to achieve conformationally restricted structures. ${ }^{[22]}$ Interestingly, the aryl-methyl-piperidine motif is found in three approved drugs as well as two of the most notable examples of RNAtargeted bioactive ligands, Ribocil B and C (Table S13). This observation indicates that incorporating conformationally restricted, multi-ring subunits on para- DPFs may be a promising future direction for the development of bioactive probes specific for the MALAT1 triple helix and potentially other RNA:ligand systems.

We were also interested in identifying the specific features that led to the shape diversity observed for the three sublibraries and thus inspected the MFE conformation from the MD simulations. First, we noted that the DPF scaffold geometry varies among the three different sublibraries. The scaffold is planar or nearly planar $\left(<6^{\circ}\right)$ for all para- substituted DPFs (Figure $3 \mathrm{~b}$, Table S12). The scaffold is non-planar for 7/11 meta-substituted DPFs, with a median furan-phenyl dihedral angle of $12^{\circ}$ (range $0-26^{\circ}$ ) (Figure $3 \mathrm{~b}$, Table S12). None of the ortho-substituted DPFs had a planar or nearly planar scaffold, and these ligands had a larger median dihedral angle of $48^{\circ}$ (range 6-88 $8^{\circ}$ ) (Figure $3 \mathrm{~b}$, Table S12). Second, we noted that the differences in scaffold dihedral angles across the three sublibraries were also marked by differences in the number and type of intramolecular interactions (Table S12). Specifically, no intramolecular interactions were observed in the para-DPF sublibrary as well as the four meta-DPFs that featured alkyl subunits, which were more rod-like and had planar or nearly planar scaffolds. On the contrary, distinct intramolecular interactions were observed for the meta-DPF ligands with aromatic subunits, which occupied disc and hybrid sub-triangles (Table S12). These included NH- $\pi$ or hydrogen bonds between the amidines and subunits as well as $\pi-\pi$ or lone pair- $\pi$ interactions between the subunits. Lastly, the ortho-DPF sublibrary members formed more intramolecular interactions, including interactions between subunits and the $\pi$-systems on the scaffold (Figure $3 \mathrm{c}$ ). For example, we observed $\mathrm{CH}-\pi$ bonds between an aliphatic subunit and the furan, as well as alternating edge-to-face and face-to-face $\pi-\pi$ interactions between the phenyl groups on the scaffold and aromatic subunits (Table S12).

Our analysis suggests that these interactions, concomitant with the increase in scaffold tilt, ultimately enable the more disc- and/or sphere-like shape of the MFE structures in the orthoand meta-DPF sublibraries. While more detailed experimental work is needed to establish the relevance of these interactions in aqueous solution, it is possible that these characteristics, which are observed with almost all subunits, lead to less variability in RNA- 
binding preferences among the sublibrary members. Further, the energetic cost of breaking intramolecular interactions may be in part responsible for the decreased binding strengths in these two sublibraries. Ultimately, we propose that these sublibraries are less likely to achieve extended, rod-like shapes that are important for selective RNA recognition. [13a]

In summary, we synthesized a DPF scaffold-based small molecule library diversified in subunit composition and positioning to explore the recognition of a newly discovered IncRNA triple helix. Library screening against MALAT1-related constructs and controls led to the discovery of the first selective ligand for the MALAT1 triple helix. This work demonstrates that a known RNA-binding scaffold can indeed be tuned to selectively recognize a unique RNA structural element by relying on subunit identity and placementbased differentiation. PMI calculations revealed striking sublibrary-based differences in small molecule shapes, a diversity deemed important for screening libraries but often difficult to achieve with scaffold-based libraries of similar size. ${ }^{[23]}$ Consistent with our previous observations, ${ }^{[13 \mathrm{a}]}$ the most promising ligands had rod-like shapes in this analysis. The more disc and sphere-like DPF ligands were predicted to have increased intramolecular interactions and scaffold dihedral angles, which generally correlated with a decrease in binding strengths for the triple helix. These characteristics were particularly prevalent in the meta- and ortho-sublibraries and may be responsible for the decrease in differential binding among these ligands. These findings will be utilized to design more extended and rod-like DPFs and expedite the discovery of chemical probes for the MALAT1 triple helix.

Furthermore, as the intramolecular interactions identified in this study formed through chemotypes that are commonly found in RNA-binding small molecules, such as aromatic and heteroatom-containing moieties, we suggest that future efforts to design RNA-targeted libraries should investigate the possibility of intramolecular interactions within ligands containing these chemotypes. ${ }^{[13 \mathrm{a}]}$ Lastly, we note that these interactions should be taken into consideration when performing docking studies to rank RNA:ligand affinities, as the analysis of energetically inaccessible conformations due to intramolecular interactions has led to dead-end designs in protein-based experiments. ${ }^{[24]}$ We hope that these novel insights and rational strategies contribute to understanding of general principles for small molecule :RNA recognition, and we anticipate that this work encourages further small molecule-based exploration of the MALAT1 triple helix and other IncRNA structural elements with emerging roles in disease biology.

\section{Supplementary Material}

Refer to Web version on PubMed Central for supplementary material.

\section{Acknowledgements}

We would like to thank all current and past members of the Hargrove lab for stimulating input and discussion. Further, we thank Dr. George Dubay for assistance with mass spectrometry instrumentation, Dr. David Gooden for assistance with the selection and optimization of synthetic methodologies, Drs. Richard Brennan and Todd Woerner for their permission to use UV/Vis instrumentation in their laboratories, and Duke Data and Visualization Services for help with figure preparation. A.D. is grateful to all members of the Al-Hashimi lab, specifically Laura Ganser, Dr. Dawn K. Merri-man, Dr. Mary Clay, Atul Kaushik Rangadurai, and Bei Liu, as well as members of the Weeks lab, specifically Dr. Natalie McDonald, for help and advice regarding RNA preparation and handling. A.E.H. wishes to acknowledge Duke University, the Prostate Cancer Foundation Young Investigator Award, and the 
National Institute of Health (NIH) Maximizing Investigator's Research Award (MIRA), Grant/Award Number: R35GM124785 for financial support. B.S.M. was supported by the Duke University Katherine Goodman Stern Fellowship, and J.L.X. was supported through the Duke University Undergraduate Research Support grant as well as the Dean's Summer Research Fellowship.

\section{References}

[1]. The Encode Project Consortium, Nature 2012, 489, 57-74. [PubMed: 22955616]

[2]. Rinn JL, Chang HY, Annu. Rev. Biochem. 2012,81,145-166. [PubMed: 22663078]

[3]. Donlic A, Hargrove AE, Wiley Interdiscip. Rev.: RNA 2018, 9, e1477. [PubMed: 29726113]

[4]. a)Ji P, Diederichs S, Wang W, Böing S, Metzger R, Schneider PM, Tidow N, Brandt B, Buerger H, Bulk E, et al., Oncogene 2003,22, 8031 - 8041; [PubMed: 12970751] b)Gutschner T, Hämmerle M, Eißmann M, Hsu J, Kim Y, Hung G, Revenko A, Arun G, Stentrup M, Groß M, et al., Cancer Res. 2013, 73,1180-1189; [PubMed: 23243023] c)Amodio N, Raimondi L, Juli G, Stamato MA, Caracciolo D, Tagliaferri P, Tassone P, J. Hematol. Oncol. 2018,11, 63. [PubMed: 29739426]

[5]. Xu C, Yang M, Tian J, Wang X, Li Z, Int. J. Oncol. 2011, 39, 169-175. [PubMed: 21503572]

[6]. a)Brown JA, Bulkley D, Wang J, Valenstein ML, Yario TA, Steitz TA, Steitz JA, Nat. Struct. Mol. Biol. 2014, 21, 633-640; [PubMed: 24952594] b)Wilusz JE, JnBaptiste CK, Lu LY, Kuhn CD, Joshua-Tor L, Sharp PA, Genes \& Dev. 2012, 26, 2392 - 2407. [PubMed: 23073843]

[7]. Xi H, Arya DP, Curr. Med. Chem. :Anti-Cancer Agents 2005,5, 327-338. [PubMed: 16101485]

[8]. a)Scaria PV, Shafer RH, J. Biol. Chem. 1991, 266, 5417-5423; [PubMed: 2005088] b)Biver T, Boggioni A, Garcia B, Leal JM, Ruiz R, Secco F, Venturini M, Nucleic Acids Res. 2010, 38, 1697-1710; [PubMed: 20008509] c)Sinha R, Kumar GS, J. Phys. Chem. B 2009, 113, 1341013420; [PubMed: 19754095] d)Lozano HJ, García B, Busto N, Leal JM, J. Phys. Chem. B 2013,117,38-48; [PubMed: 23206123] e)García B, Leal JM, Paiotta V, Ruiz R, Secco F, Venturini M, J. Phys. Chem. B 2008,112, 7132-7139. [PubMed: 18491933]

[9]. a)Zhao M, Ratmeyer L, Peloquin RG, Yao S, Kumar A, Spychala J, Boykin DW, Wilson WD, Bioorg. Med. Chem. 1995, 3, 785-794; [PubMed: 7582956] b)Yang WY, Gao R, Southern M, Sarkar PS, Disney MD, Nat. Commun. 2016, 7, 11647. [PubMed: 27248057]

[10]. a)Chaires JB, Ren J, Hamelberg D, Kumar A, Pandya V, Boykin DW, Wilson WD, J. Med. Chem. 2004, 47, 5729-5742. [PubMed: 15509172]

[11]. Gelus N, Bailly C, Hamy F, Klimkait T, Wilson WD, Boykin DW, Bioorg. Med. Chem. 1999, 7, 1089-1096; [PubMed: 10428378] b)Ratmeyer L, Zapp ML, Green MR, Vinayak R, Kumar A, Boykin DW, Wilson WD, Biochemistry 1996, 35, 13689-13696; [PubMed: 8885849] c)Zapp ML, Young DW, Kumar A, Singh R, Boykin DW, Wilson WD, Green MR, Bioorg. Med. Chem. 1997, 5, 1149-1155. [PubMed: 9222509]

[12]. Fu HY, Doucet H, Eur. J. Org. Chem. 2011, 7163-7173.

[13]. a)Morgan BS, Forte JE, Culver RN, Zhang Y, Hargrove AE, Angew. Chem. Int. Ed. 2017, 56, 13498-13502; Angew. Chem. 2017, 129, 13683-13687;b)Vourloumis D, Takahashi M, Simonsen KB, Ayida BK, Barluenga S, Winters GC, Hermann T, Tetrahedron Lett. 2003, 44, 2807 2811 ; c)Velagapudi SP, Pushechnikov A, Labuda LP, French JM, Disney MD, ACS Chem. Biol. 2012, 7, 1902-1909. [PubMed: 22958065]

[14]. Rousselet G, Capdevielle P, Maumy M, Tetrahedron Lett 1993, 34, 6395-6398.

[15]. Lee DS, Amara Z, Poliakoff M, Harman T, Reid G, Rhodes B, Brough S, Mclnally T, Woodward S, Org. Process Res. Dev. 2015,19, 831-840.

[16]. Boykin DW, Kumar A, Spychala J, Zhou M, Lombardy RJ, Wilson WD, Dykstra CC, Jones SK, Hall JE, Tidwell RR, et al., J. Med. Chem. 1995, 38, 912-916. [PubMed: 7699707]

[17]. Xiao G, Kumar A, Li K, Rigl CT, Bajic M, Davis TM, Boykin DW, Wilson WD, Bioorg. Med. Chem. 2001, 9, 1097-1113. [PubMed: 11377168]

[18]. Brown JA, Valenstein ML, Yario TA, Tycowski KT, Steitz JA, Proc. Natl. Acad. Sci. USA 2012,109, 19202-19207. [PubMed: 23129630]

[19]. Nguyen B, Tardy C, Bailly C, Colson P, Houssier C, Kumar A, Boykin DW, Wilson WD, Biopolymers 2002, 63, 281-297. [PubMed: 11877739]

Angew Chem Int Ed Engl. Author manuscript; available in PMC 2019 October 01. 
[20]. Charrette BP, Boerneke MA, Hermann T, ACS Chem. Biol. 2016,11, 3263-3267. [PubMed: 27775338]

[21]. a)Sauer WH, Schwarz MK, J. Chem. Inf Comput. Sci. 2003, 43, 987-1003; [PubMed: 12767158] b)Wirth M, Sauer WH, Mol. Inform. 2011, 30, 677 - 688. [PubMed: 27467260]

[22]. Fang Z, Song Y, Zhan P, Zhang Q, Liu X, Future Med. Chem. 2014, 6, 885-901. [PubMed: 24962281]

[23]. Galloway WR, Isidro-Llobet A, Spring DR, Nat. Commun. 2010,1, 80. [PubMed: 20865796]

[24]. Hudson BM, Nguyen E, Tantillo DJ, Org. Biomol. Chem. 2016,14, 3975-3980. [PubMed: 27049933] 
a)

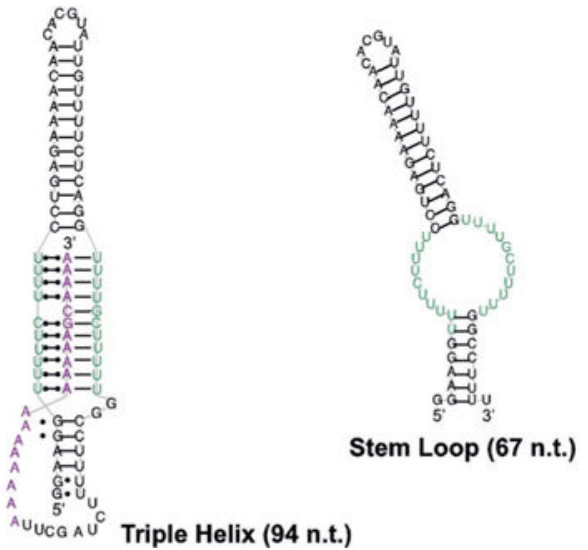

b)

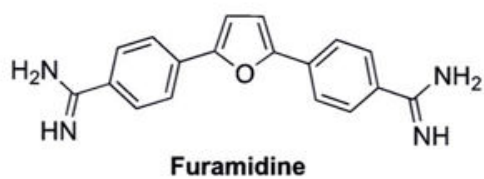

c)

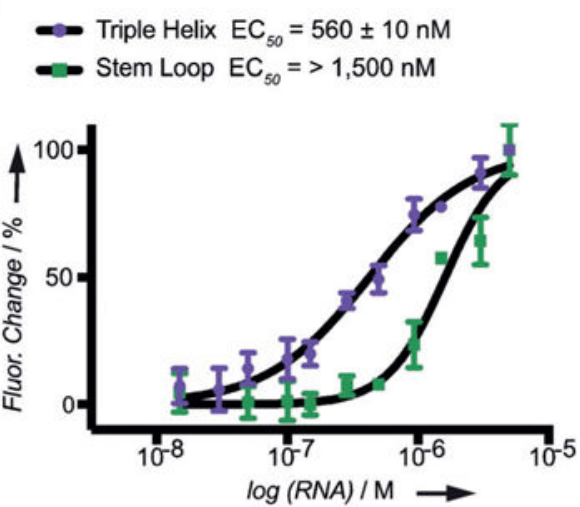

Figure 1.

a) MALAT1 triple helix (94 nucleotides, left) and non-triplex-forming stem loop (67 nucleotides, right) constructs; n.t. $=$ nucleotide. b) Structure of furamidine. c) Normalized fluorescence change of furamidine $(1 \mu \mathrm{m})$ upon titration of 0.015 to $5 \mu \mathrm{m}$ triple helix (purple) and stem loop RNA (green). $\mathrm{EC}_{50}$ values were determined from triplicate data using the change in fluorescence intensity. No saturable binding was observed for values over 1.5 $\mu \mathrm{m}$, resulting in ambiguous curve fits. Error bars represent the standard deviation determined from three independent measurements. 

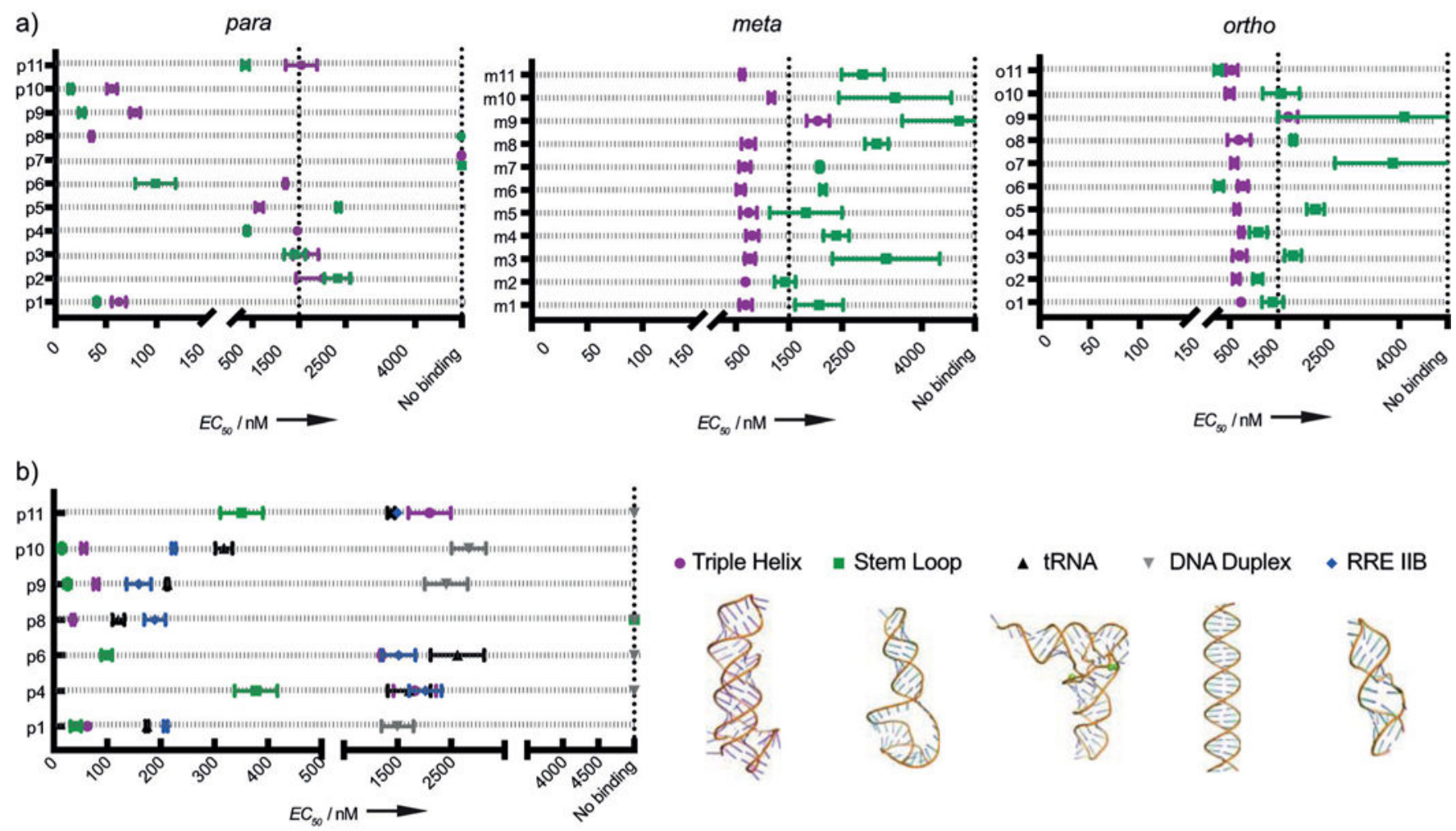

Figure 2.

a) $\mathrm{EC}_{50}$ values of the para-(left), meta- (center) and ortho-(right) sublibraries towards the MALAT1 triple helix (purple, PDB I.D.: 4PLX) and stem loop (green) constructs. b) Selectivity screen of select para-sublibrary members against yeast tRNA (76-90 n.t., PDB I.D.:4TNA) (black), DNA duplex (28 n.t.) (gray) and RRE Stem Loop IIB (34 n.t.) (blue). $\mathrm{EC}_{50}$ values were determined from triplicate data using the change in fluorescence intensity. First dashed line indicates measurements above $1500 \mathrm{nM}$ for which no saturable binding events were observed, resulting in ambiguous curve fits. No binding indicates no change in fluorescence intensity of the DPF ( $1 \mu \mathrm{m}$ ) upon RNA/DNA titration (up to $5 \mu \mathrm{m}$ ). Error bars represent the standard deviation determined from three independent measurements. 
a)

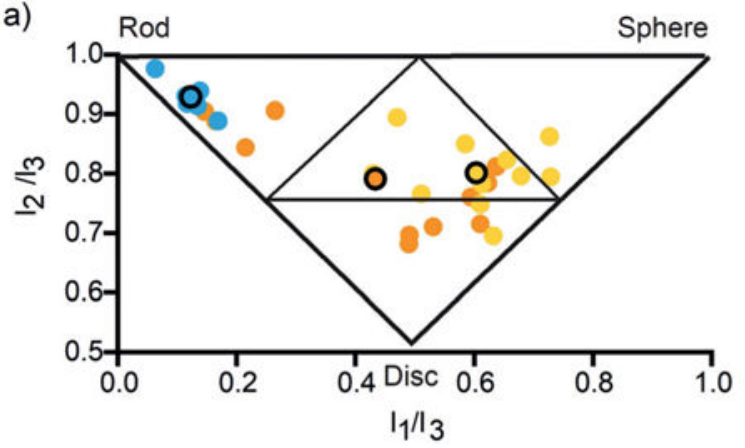

- Para AVG

O Ortho AVG

O Meta AVG

- Para

- Ortho

- Meta

b)

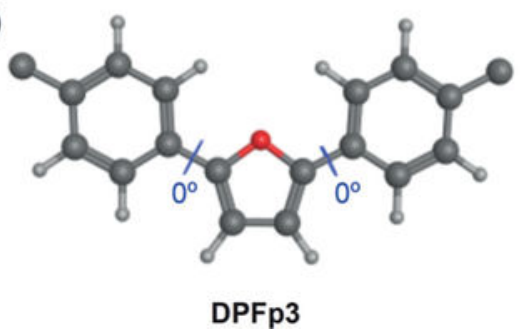

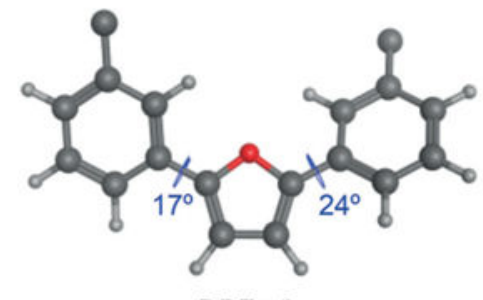

DPFm3 c)
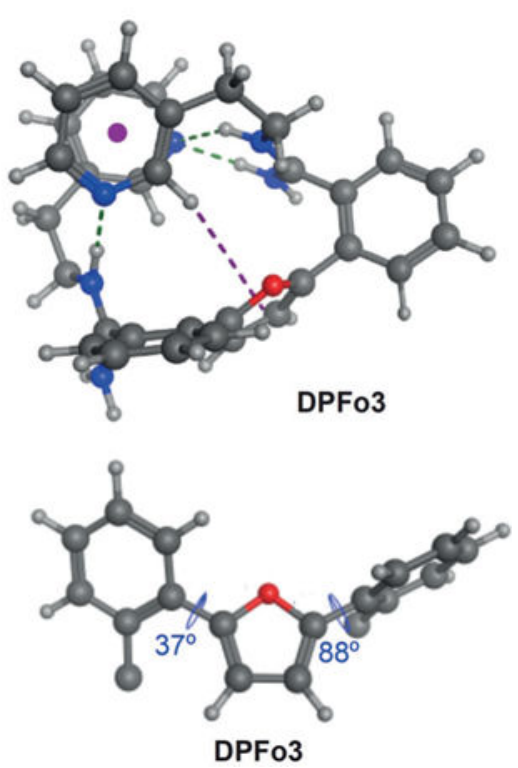

Figure 3.

a) Principal moments of inertia (PMI) calculations of the DPF library. AVG: average. b) Minimal free energy (MFE) structures of example ligands: DPFp3 (left), DPFm3 (center) and DPFo3 (right) with furan-phenyl dihedral angles indicated. Circles (blue) indicate the dihedral angles between the furan oxygen and the contiguous phenyl ring carbon atoms. Subunits and amidine nitrogens were omitted for clarity. c) MFE of DPFo3 with intramolecular interactions: hydrogen bonds (green line), edge-to-face $\pi-\pi$ (purple line), and face-to-face $\pi-\pi$ (purple dot). 


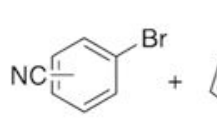

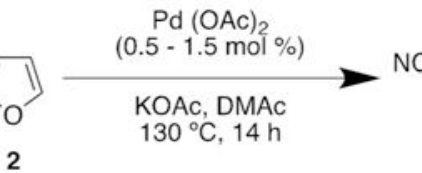

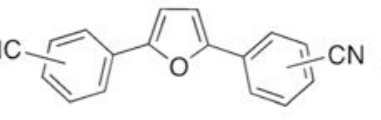

3

$47 \%, \mathrm{CN}=$ para $41 \%, \mathrm{CN}=$ meta $60 \%, \mathrm{CN}=$ ortho

$\mathbf{R}=$
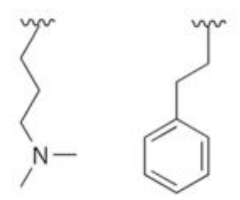<smiles>CCCc1cccnc1</smiles><smiles>CCc1ccccc1</smiles><smiles>CCc1ccc(Cl)cc1</smiles><smiles>CCc1ccccc1Cl</smiles>

$\begin{array}{ll}p 1,7 \% & p 2,19 \% \\ m 1,9 \% \text { a } & m 2,46 \%\end{array}$ $m 1,9 \%$ a $m 2,46 \%$ p3, $11 \%$
m3, $8 \%$ $03,44 \%$

p $4,78 \%$ $m 4,68 \%$ $04,72 \%$

$p 5,59 \%$
$m 5,39 \%$ $05,42 \%$ p6, $83 \%$ $m 6,48 \%$ $06,30 \%$

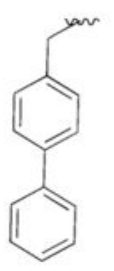

p7, $19 \%$ $m 7,50 \%$ o7, $24 \%$
R- $\mathrm{NH}_{2}, \mathrm{CuCl}$

$\mathrm{EtOH}$, reflux, $48 \mathrm{~h}$

OR

R-NH ${ }_{2}, \mathrm{DABAL}-\mathrm{Me}_{3}$

$\mathrm{THF}, \mu \mathrm{W}, 100^{\circ} \mathrm{C}$ $1.5-8 \mathrm{~h}$

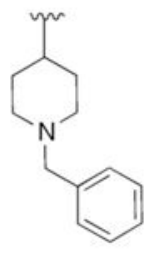<smiles>CCCN1CCNCC1</smiles><smiles>CCCN1CCN(C)CC1</smiles><smiles>CCCc1ccsc1</smiles>

p8, $9 \%$ o8, $39 \%$

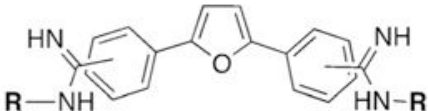

DPF-R

Scheme 1.

Synthesis of the diphenylfuran-based small molecule library, [a] Lower final yield was due to difficulty in purifying highly polar compounds by normal phase column chromatography. 